\title{
Diseño, modelado e implementación de inversor conectado a la red eléctrica a partir de fuentes renovables
}

\section{A renewable-source-based inverter pluged to the electrical grid - design, modelling and implementation}

César Leonardo Trujillo Rodríguez

Ingeniero Electrónico, magister en Ingeniería Eléctrica, Doctor en Ingeniería Electrónica. Docente Asociado de la Universidad Distrital Francisco José de Caldas. Bogotá, Colombia.cltrujillo@udistrital.edu.co.

David Velasco de la Fuente

Ingeniero Industrial, magister en Sistemas Electrónicos de Potencia. Investigador de la Universidad Politécnica de Valencia. Valencia, España.davede@upv.es

\section{Emilio Figueres Amorós}

Ingeniero Técnico Industrial, doctor en Ingeniería Industrial. Docente Catedrático de la Universidad Politécnica de Valencia. Valencia, España.efiguere@eln. upv.es

\section{Gabriel Garcerá Sanfeliú}

Ingeniero en Telecomunicación, doctor Ingeniero de Telecomunicación. Docente Catedrático de la Universidad Politécnica de Valencia. Valencia, España. ggarcera@eln.upv.es

\section{Javier Guacaneme Moreno}

Ingeniero Electrónico, magister en Ingeniería Eléctrica. Docente Asociado de la Universidad Distrital Francisco José de Caldas. Bogotá, Colombia. jguacaneme@udistrital.edu.co

Clasificación del artículo: Investigación (Conciencias)

Fecha de recepción: Agosto 10 de 2011

Fecha de aceptación: Febrero 27 de 2012

Palabras clave: control de corriente, generación de potencia distribuida, inversor.

Key words: power control, distributed power generation, investor. 


\section{con-ciencias}

\section{RESUMEN}

En este artículo se describe el diseño, modelado e implementación de un inversor monofásico conectado a la red a partir de fuentes renovables de energía. Se estudia el modelo en pequeña señal del convertidor para luego diseñar los lazos de control del mismo. Con el fin de controlar la inyección de energía a la red proveniente de la renovable, se propone utilizar un control de corriente media (ACC). Los controladores son obtenidos a través del análisis aplicado sobre el modelo en pequeña señal obtenido a partir del modelo del conmutador PWM y de la aproximación del inversor a un convertidor Buck. Finalmente, se realizan simulaciones y pruebas experimentales con el fin de validar la respuesta transitoria y en estado estable del inversor.

\section{ABSTRACT}

This paper describes the design, modeling and implementation of a single-phase inverter connected to the grid from renewable energy sources. We study the model in small signal to be below the control loops design it. In order to control the injection of energy into the grid from renewable is proposed for average current control (ACC). Drivers are obtained through the analysis applied to the small signal model obtained from the PWM switch model and the approach of the investor to a Buck converter. Finally, simulations and experimental tests conducted to validate the transient response and steady-state investor

\section{INTRODUCCIÓN}

En la actualidad, temas ambientales como el calentamiento global y la polución son cada vez más relevantes para la sociedad, por otra parte los gobiernos día tras día ponen más objeciones a la utilización de las centrales eléctricas convencionales y nucleares. En consecuencia, el uso de fuentes alternativas de energía tales como la energía eólica, la energía solar, la biomasa renovable y el hidrógeno se perfilan como una solución viable para la producción de energía limpia [1].

Debido a las propiedades de la energía captada a partir de las fuentes renovables se hace necesaria la utilización de sistemas electrónicos, convertidores, que permitan adaptar las condiciones impuestas por la carga. La energía procedente de las fuentes renovables se puede obtener en forma de corriente continua como es el caso de la energía fotovoltaica [2], y en forma de corriente alterna con frecuencia variable en el caso de la energía eólica [3]. Por esta razón, la solución más habitual es partir de corriente continua o alterna y convertirla a corriente alterna con las mismas características eléctricas de la red mediante la utilización de inversores. Estos inversores deben operar como una fuente de corriente en fase con la tensión de red, inyectando potencia a la red de distribución [4], [5]. En consecuencia, la idea del artículo es presentar el diseño, modelado e implementación de un inversor interconectado a la red eléctrica a partir de fuentes renovables.

El artículo se ha esquematizado de la siguiente manera: en la sección 1 se hace un análisis de funcionamiento del inversor monofásico implementado. En la sección 2, se presenta el modelado dinámico en pequeña señal del inversor. En la sección 3, se presenta el diseño e implementación de los controladores para el ACC. En la sección 4 y la sección 5 se presentan los resultados de simulación y los resultados experimentales del inversor, respectivamente. Se finaliza con las conclusiones 


\section{ANÁLISIS DE FUNCIONAMIENTO DEL INVERSOR MONOFÁSICO}

El inversor monofásico con topología de puente completo y empleando un esquema de Modulación por Ancho de Pulso (PWM) ha sido ampliamente usado en Sistemas de Alimentación Ininterrumpida (UPS) [6], como interfaz DC/AC de fuentes no convencionales de energía, tales como el viento [7] y el sol [8], en reguladores de tensión [9] y en muchas otras aplicaciones industriales.

Esta topología permite obtener sobre la carga cuya tensión en la Fig. 1 se identifica como Vab, tensiones positivas, negativas o cero, según lo determine el circuito de control de los interruptores [10], [11]. La única precaución que hay que tener en cuenta al generar los disparos, es que dos interruptores de una misma rama (S1, S3 o S2, S4) no deben ser encendidos al mismo tiempo ya que se originaría un cortocircuito alrededor de la fuente DC. Adicionalmente se necesitan diodos en antiparalelo para garantizar la continuidad de la corriente en presencia de cargas inductivas. En la Fig. 1 se muestra un esquema del inversor puente completo

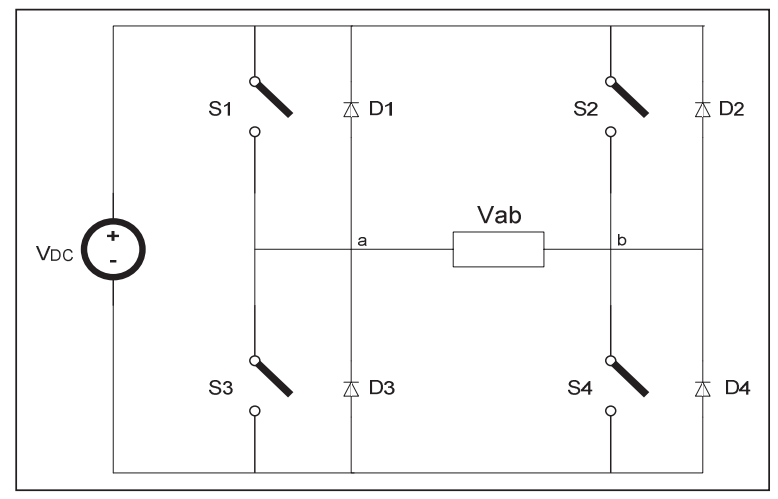

Fig. 1. Esquema del inversor a puente completo

\subsection{Diseño del filtro de conexión a red}

Einversor se debe diseñar para una potencia nominal de 430VA, una tensión de salida de

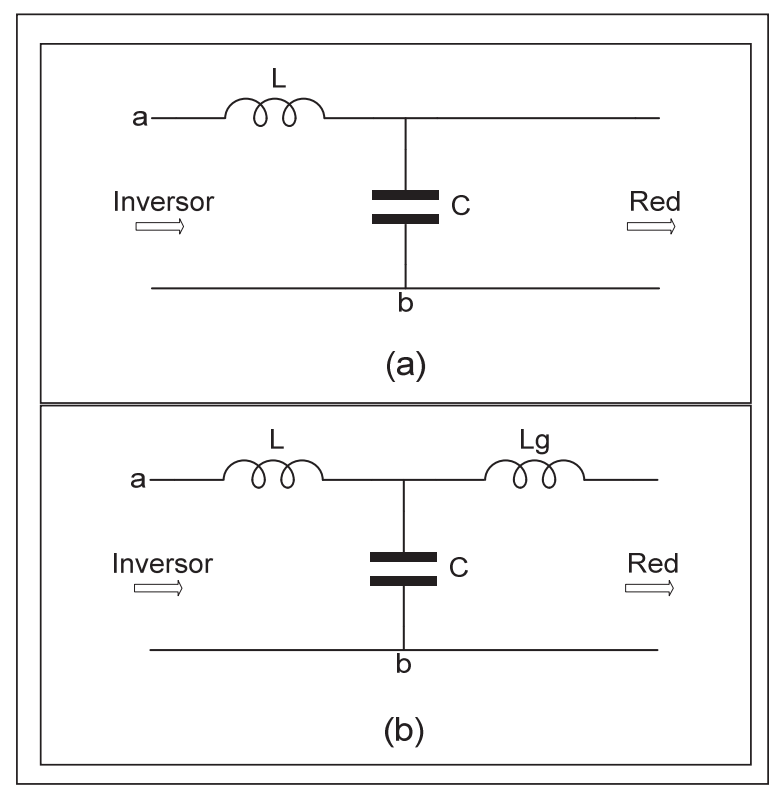

Fig. 2. Filtro (a) LC, (b) LCL

230VRMS, frecuencia de $50 \mathrm{~Hz}$ para la moduladora y de $20 \mathrm{kHz}$ para la portadora, la cual presenta forma de onda triangular, y para un esquema de conmutación PWM bipolar. Dicho esquema de modulación se seleccionó debido a que permite reducir las corrientes en modo común (CMC) [12], contrario a lo que se presenta con la modulación unipolar.

Con el fin de obtener una señal de salida sinusoidal con las mismas características en tensión y frecuencia que la de la red eléctrica, es necesario filtrar la tensión $V_{a b}$ presente entre las ramas del inversor monofásico en puente completo. Las topologías de filtros de salida comúnmente empleadas en inversores son la LC y la LCL [13] - [15]. En la Fig. 2 se muestra las topologías circuitales de los filtros de salida del inversor.

El filtro seleccionado para esta aplicación fue el filtro LCL el cual presenta una atenuación de $60 \mathrm{db} /$ década a partir de la frecuencia de resonancia, tiene una baja distorsión de la corriente de red y baja producción de potencia reactiva, sin embargo, puede llegar a causar distorsión de la 


\section{con-ciencias}

corriente de entrada, dinámica y en estado estacionario, debido a la resonancia [16].

Algunas características que debe presentar el filtro a la hora de ser diseñado son las siguientes:

El filtro LCL se puede ver como un filtro LC más una inductancia de fugas que en muchas ocasiones puede ser tratada como la inductancia de fugas del transformador de aislamiento.

A la frecuencia fundamental, el condensador debe absorber poca potencia reactiva del fundamental, con lo que la corriente en el inductor conectado del lado del inversor apenas se ve incrementada por el condensador.

A la frecuencia de los armónicos de conmutación, el condensador debe absorber los armónicos de la corriente del inductor los cuales deben ser pequeños.

Con base en lo anterior se describirá el procedimiento para determinar los valores de dicho filtro.

El valor del condensador $C$, está limitado al consumo máximo de la potencia reactiva permitido por el inversor.

Para este caso si se requiere que la corriente máxima en el condensador $\left(I_{C}\right)$ sea inferior al $5 \%$ de la corriente nominal $\left(I_{O}\right)$, se tiene en magnitud:

$$
I_{C}=0,05 \cdot I_{O}
$$

Por tanto, la magnitud de la impedancia del condensador será la tensión de salida partida por la corriente en el condensador

$$
Z_{C}=\frac{V_{o}}{I_{C}}=\frac{V_{o}}{0.05 \cdot\left(\frac{S_{O}}{V_{o}}\right)}=2404,5 \Omega
$$

Siendo $S_{O}$, la potencia nominal de la carga. Como la magnitud de $Z c=1 / \omega_{1} \cdot C$ se puede despejar el valor de $C$.

$$
C=\frac{1}{\omega_{1} \cdot Z_{C}} \leq 1,3 \mu \mathrm{F}
$$

Donde $\omega_{1}$ es la frecuencia de la red en $\mathrm{rad} / \mathrm{s}$.

El inductor del lado del inversor se puede calcular en términos de la caída de tensión permitida sobre este, cuando la tensión a la salida del inversor sea la de operación normal.

Asumiendo que se quiere una impedancia del inductor igual o menor que el 5\% de la impedancia nominal de la carga, se tiene

$$
Z_{L} \leq 0,05 \cdot Z_{L O A D}
$$

La magnitud de la impedancia del inductor se define como $Z L=\omega_{1} \cdot L$. Despejando el valor de $L$ se tiene:

$$
L=\frac{Z_{L}}{\omega_{1}}=\frac{0.05 \cdot Z_{L O A D}}{\omega_{1}} \leq 19,1 m H
$$

La frecuencia de resonancia del filtro LCL se debe localizar entre 10 veces la frecuencia de red y la mitad de la frecuencia de conmutación, esto con el fin de evitar problemas de resonancia en la parte baja y alta del espectro armónico [15].

$$
10 \cdot \omega_{1}<\omega_{r e s}<\frac{\omega_{S}}{2}
$$

Donde $\omega_{\text {res }}$ es la frecuencia de resonancia del filtro LCL, la cual se define por Ec. (7) y $\omega_{s}$ la frecuencia de conmutación.

$$
\omega_{r e s}=\sqrt{\frac{L+L_{g}}{L \cdot L_{g} \cdot C}}
$$




\section{con-ciencias}

Con el fin de cumplir la condición 3) así como la condición 1) se tomará un valor de $\mathrm{C}=600 \mathrm{nF}$ y se considerará que la inductancia de red $L_{g}$ es diez veces menor al valor hallado para el inductor conectado al inversor, sin embargo, es de aclarar que dicho valor depende de la suma de la inductancia de los cables más la inductancia del transformador, la cual puede variar dependiendo de la localización del inversor.

Los filtros pasivos LC o LCL presentan un alto factor de calidad $Q_{f}$, por tanto muestran una baja amortiguación a la frecuencia de resonancia que puede causar inestabilidad en el sistema. Una forma de aumentar el amortiguamiento es agregando una resistencia en serie con el condensador. Se debe tener en cuenta que el seleccionar una resistencia $R_{d}$, muy grande reducirá notablemente la oscilación a la frecuencia de resonancia al igual que la eficiencia del sistema [15], [16]. Un criterio adicional que puede ser incluido, se basa en colocar una resistencia que permita que la atenuación de la oscilación del lazo de control de corriente del inversor esté por debajo de los 0dB. Por lo tanto el valor de $R_{d}$ será calculado cuando se aborden los lazos de control.

\subsection{Diseño del bus de continúa}

El valor del condensador del bus te continúa o DC_LINK (Ver Fig. 3) puede ser expresado como [17]:

$$
C_{D C_{-} L I N K}=\frac{P_{\text {nom }}}{2 \cdot \omega \cdot V_{D C} \cdot \Delta V_{D C}}
$$

Donde, Pnom es la potencia nominal de entrada al inversor, proveniente de la fuente primaria (paneles fotovoltaicos, convertidor AC/DC conectado a una turbina eólica, etc.), $V_{D C}$ es la tensión media alrededor del condensador y $\Delta V_{D C}$ el rizado de la tensión permitido en el condensador

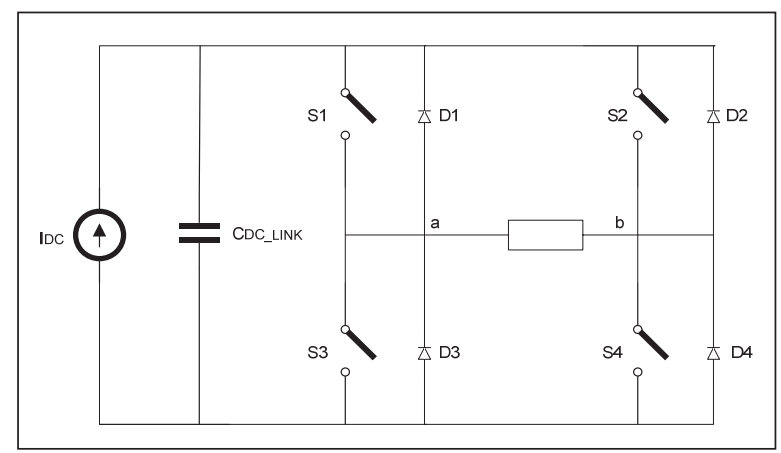

Fig. 3. Esquema del inversor a puente completo con CDC_LINK

(1\%). Dicha ecuación se basa en el hecho de que la corriente que proviene de la fuente primaria es una DC y la corriente de entrada al inversor sigue la forma de onda $\sin ^{2}(\omega \bullet t)$. Con lo que se obtiene un valor de,

$$
C_{D C_{-} L I N K} \geq 437,68 U F
$$

Una característica adicional al elegir el valor de $C_{D C_{-} L I N K}$, se basa en el valor del rizado de corriente a $100 \mathrm{~Hz}$ y la de corriente RMS, que debe soportar dicho condensador, la cual se encuentra definida para modulación bipolar como [18]:

$$
I_{C_{D C_{L} L N K} R M S}=I_{p} \cdot \sqrt{\frac{1}{8}\left[4-m_{m}^{2} \cdot\left(1+2 \cdot \cos ^{2}\left(\varphi_{p}\right)\right)\right]} \cong 1.5 \mathrm{~A}
$$

Donde $I_{p}$ es el valor pico de la corriente, $\varphi_{p}$ el ángulo de fase entre la tensión y la corriente de salida del inversor y $\mathrm{mm}$ el factor de modulación. El valor de corriente hallado anteriormente (1.5A), así como el rizado de corriente a $100 \mathrm{~Hz}$ (6A) permite identificar según las especificaciones técnicas del fabricante, si el valor de condensador elegido soporta dichos valores de corriente para una tensión DC determinada, de no ser así se toma un valor de capacitancia superior que cumpla con las condiciones de corriente y tensión. El valor de $\mathrm{C}_{\mathrm{DC} \text { LINK }}$ para esta aplicación según la hoja de especificaciones es de $1 \mathrm{mF}$ 


\section{MODELADO DINÁMICO EN PEQUEÑA SEÑAL DEL INVERSOR}

Debido a que los diferentes convertidores (DC/ DC o DC/AC) son circuitos no lineales, estos deben ser linealizados en torno a un punto de operación con el fin de poder aplicar técnicas de control lineal. La técnica seleccionada para linealizar la etapa de potencia es la del modelo del conmutador PWM [19], [20]. Este modelo ofrece un circuito equivalente lineal, a partir del cual se puede realizar el análisis de pequeña señal y de gran señal del convertidor. El método se basa en la sustitución de los elementos no lineales del convertidor conmutado (interruptores), por un circuito equivalente lineal llamado "Modelo del Conmutador PWM", de manera que el circuito resultante es lineal.

En la Fig. 4 se presenta el esquema circuital del inversor, sobre el cual se obtendrá el modelo.

El inversor puede ser aproximado a un circuito Buck [11], teniendo en cuenta que en el punto de operación se manejan señales periódicas variantes en el tiempo. La ecuación que relaciona la tensión de salida con la tensión de entrada en términos del ciclo de trabajo se muestra en Ec. (11).

$$
V_{O}=V_{D C} \cdot(2 \cdot D-1)
$$

Si se considera que cada una de las variables promediadas se puede descomponer en un término constante correspondiente al punto de trabajo (en mayúsculas) y en un término variante de pequeña señal (con " $\wedge$ ") que representa la perturbación en torno al punto de trabajo, la ecuación anterior toma el siguiente aspecto

$$
V_{O}+\hat{v}_{O}=\left(V_{D C}+\hat{v}_{D C}\right) \cdot(2 \cdot(D+\hat{d})-1)
$$

Separando las componentes constantes y variables se tiene:

$$
\begin{gathered}
V_{O}=V_{D C} \cdot(2 \cdot D-1) \\
\hat{v}_{O}=\hat{v}_{D C} \cdot(2 \cdot D-1)+2 \cdot V_{D C} \cdot \hat{d}
\end{gathered}
$$

De la misma forma se pueden obtener las componentes constantes y variables para la relación entre la corriente de entrada y la corriente de salida

$$
\begin{gathered}
V_{O}=V_{D C} \cdot(2 \cdot D-1) \\
\hat{v}_{O}=\hat{v}_{D C} \cdot(2 \cdot D-1)+2 \cdot V_{D C} \cdot \hat{d}
\end{gathered}
$$

Con base en la Ec. (13) y la Ec. (14) es posible obtener el modelo en el punto de operación (Fig. 5) y el modelo de pequeña señal (Fig. 6) del inversor.

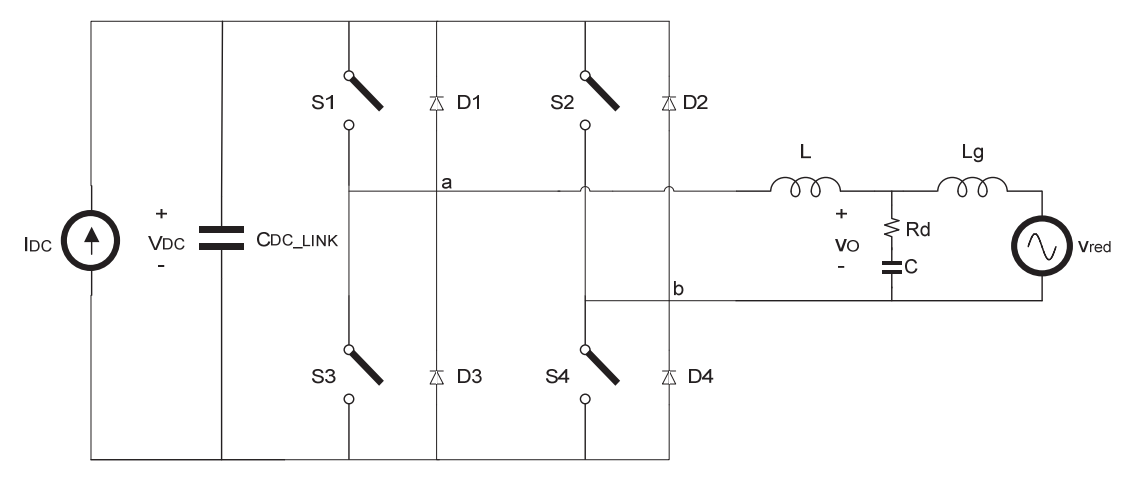

Fig. 4. Esquema circuital del Inversor. Esquema circuital del Inversor 


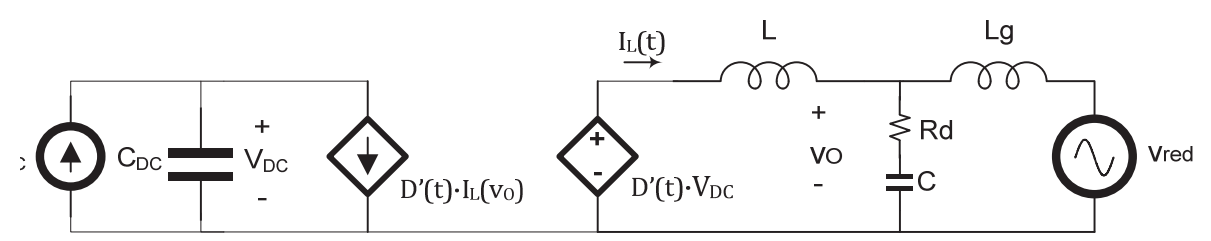

Fig. 5. Modelo equivalente del punto de operación del inversor

Donde $D^{\prime}(t)=2 \cdot D(t)-1$, siendo $D(t)$ el ciclo de trabajo en el punto de operación.

Las relaciones establecidas para el punto de operación se presentan en las siguientes ecuaciones:

$I_{L}(t)=\frac{\sqrt{2} \cdot P_{0}}{V_{\text {red_RMS }}} \cdot \cos (\omega t)$
$\frac{1}{2}+\frac{V_{\text {red_RMS }}^{2}+\left(\frac{\omega \cdot L \cdot P_{0}}{V_{\text {red_RMS }}}\right)^{2}}{\sqrt{2} V_{\text {DC }}} \cdot \cos \left(\omega t+a \tan \left(\frac{\omega \cdot L \cdot P_{0}}{V_{\text {red_RMS }}^{2}}\right)\right)$

Donde $\omega t$ es una variable que permite generar un barrido paramétrico con el fin de observar el comportamiento del inversor, para el punto de operación cuando la corriente y el ciclo de trabajo varían en función de la tensión de salida. En una primera aproximación $\omega t$ será igual a cero.

El modelo en pequeña señal se muestra en la Fig. 6.

\section{METODOLOGÍA}

El esquema de control utilizado para el inversor es el control de corriente media (ACC) [21]. Mediante dicho esquema se controla la corriente media en el inductor de salida, la cual será inyectada en la red eléctrica. La segunda variable de control corresponde a la tensión de entrada del inversor (DC_LINK). En la Fig. 7 se muestran los lazos de corriente y tensión del ACC para el inversor

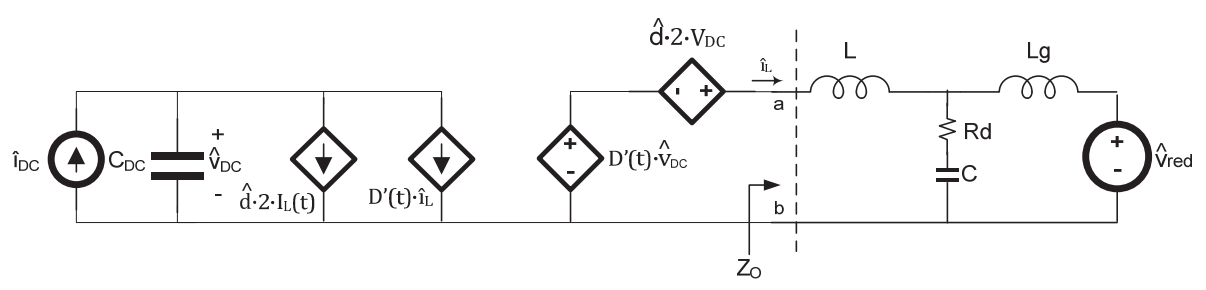

Fig. 6. Modelo de pequeña señal del inversor

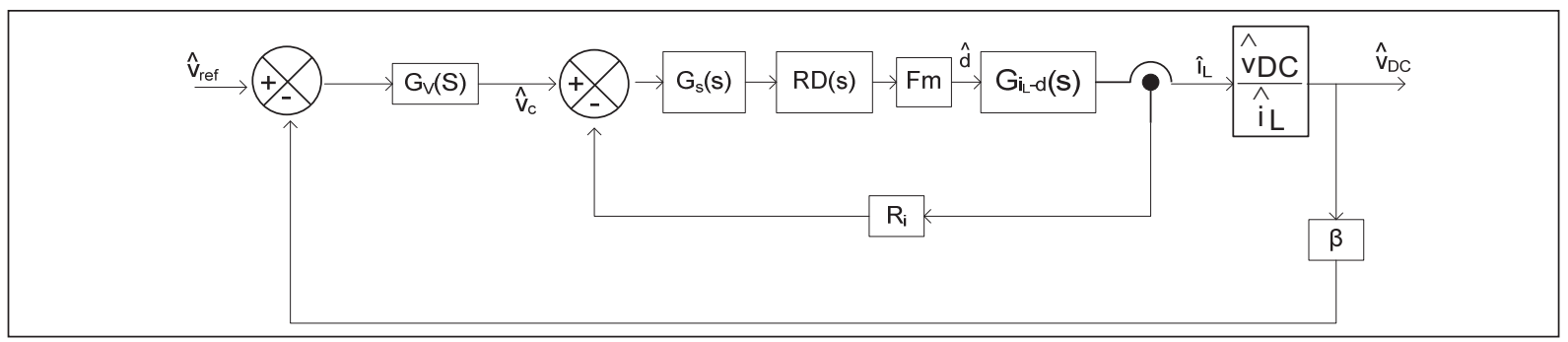

Fig. 7. Lazos de control de tensión y corriente del ACC para el inversor. 
en este modo de operación. Por simplicidad de la figura se ha omitido el PLL.

Las funciones de transferencia en lazo abierto de la etapa de potencia que relacionan la tensión de entrada (DC_LINK) y el ciclo de trabajo $\left(G_{V D C-d}(s)\right)$, y la corriente en el inductor de salida y el ciclo de trabajo $\left(G_{i L-d}(s)\right)$, se pueden extraer de los modelos dinámicos planteados en la Fig. 5 y la Fig. 6. Dichas funciones de transferencia se presentan a continuación:

Para obtener la función de transferencia $G_{V D C-d}(s)$ se tiene que

$$
G_{V D C-d}(s)=\left.\frac{\hat{v}_{D C}}{\hat{d}}\right|_{\hat{i_{D C}=v_{O}=0}}
$$

Teniendo en cuenta la Ec. (16), y aplicando análisis de nodos y análisis de mallas al circuito de la Fig. 6 se obtiene el siguiente grupo de ecuaciones:

$$
\begin{gathered}
s \cdot C_{D C} \cdot \hat{v}_{D C}+2 \cdot I_{L}(t) \cdot \hat{d}+D^{\prime}(t) \cdot \hat{i}_{L}=0 \\
D^{\prime}(t) \cdot \hat{v}_{D C}+2 \cdot V_{D C} \hat{d}=\hat{i}_{L} \cdot Z_{O}
\end{gathered}
$$

De donde $Z_{O}$ es la impedancia vista en los puntos a-b y se define como:

$$
Z_{O}=\frac{s^{3} \cdot L \cdot L_{g} \cdot C+s^{2} \cdot\left(L \cdot C \cdot R_{d}+L_{g} \cdot C \cdot R_{d}\right)+s \cdot\left(L+L_{g}\right)}{s^{2} \cdot L_{g} \cdot C+s \cdot C \cdot R_{d}+1}
$$

Despejando $\hat{\imath}_{L}$ de la Ec. (18)

$$
\hat{i}_{L}=\frac{D^{\prime}(t) \cdot \hat{v}_{D C}+2 \cdot V_{D C} \cdot \hat{d}}{Z_{O}}
$$

Reemplazando la Ec. (20) en la Ec. (17) y hallando la función de transferencia correspondiente se tiene:

$$
G_{V D C-d}(s)=\left.\frac{\hat{v}_{D C}}{\hat{d}}\right|_{\hat{i}_{D C=\hat{v}_{0}=0}}=-\frac{\left(Z_{O} \cdot 2 \cdot I_{L}(t)+D^{\prime}(t) \cdot 2 \cdot V_{D C}\right)}{s \cdot C_{D C} \cdot Z_{O}+D^{\prime}(t)^{2}}
$$

La segunda función de transferencia es

$$
G_{i L-d}(s)=\left.\frac{\hat{i}_{L}}{\hat{d}}\right|_{\hat{i}_{D C}=\hat{v}_{O}=0}
$$

Dicha función de transferencia se puede determinar a partir de la Ec. (16) y la Ec. (17) halladas anteriormente.

Por lo tanto despejando $\hat{\text { vDC }}$ de la Ec. (18)

$$
\hat{v}_{D C}=-\frac{\hat{i}_{L} \cdot Z_{O}-2 \cdot V_{D C} \cdot \hat{d}}{D^{\prime}(t)}
$$

Reemplazando la Ec. (23) en la Ec. (17) y hallando la función de transferencia correspondiente se tiene:

$$
G_{i L-d}(s)=\left.\frac{\hat{i_{L}}}{\hat{d}}\right|_{\hat{i}_{D C=v_{O}=0}}=\frac{s \cdot C_{D C} \cdot 2 \cdot V_{D C}-2 \cdot I_{L}(t) \cdot D^{\prime}(t)}{s \cdot C_{D C} \cdot Z_{O}+D^{\prime}(t)^{2}}
$$

Usando los valores obtenidos para el inversor, y variando la resistencia $R_{d}$ entre $1 \Omega$ y $50 \Omega$, se obtienen los diagramas de bode de $G_{V D C-d}(s)$ y $G_{i L-}$ ${ }_{d}(s)$, mostrados en la Fig. 8 y la Fig. 9.

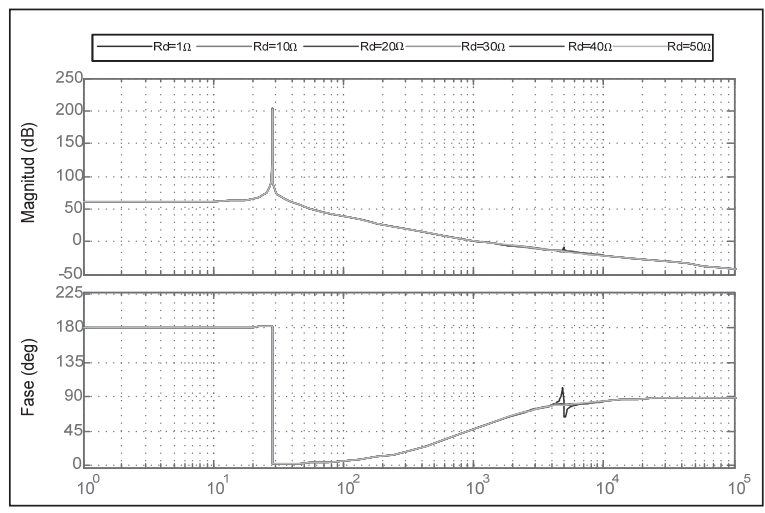

Fig. 8. Diagrama de bode de la función de transferencia $G_{V D C-d}(s)$ con variación de $R_{d}$. 


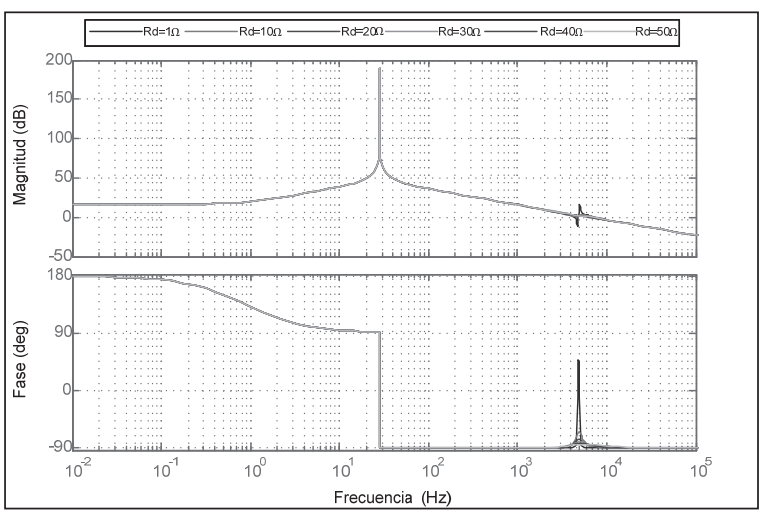

Fig. 9. Diagrama de bode de la función de transferencia $G_{i L-d}$ con variación de $R_{d}$.

Se puede observar que cuanto mayor sea el valor de $R_{d}$ se tendrá un mayor amortiguamiento producto de la resonancia en el filtro de salida, sin embargo un valor muy grande puede reducir la eficiencia.

Una vez obtenidas las funciones de transferencia, se procederá a determinar el retardo digital $(\mathrm{RD}(\mathrm{s}))$ de un periodo de conmutación, que para este caso se ha expresado como una aproximación de Tustin de segundo orden.

$$
R D(s)=\frac{1-\left(\frac{s \cdot T_{s}}{2}\right)+\left(\frac{\left(s \cdot T_{s}\right)^{2}}{12}\right)}{1+\left(\frac{s \cdot T_{s}}{2}\right)+\left(\frac{\left(s \cdot T_{s}\right)^{2}}{12}\right)}
$$

De donde $T s=50 \mu \mathrm{s}$ es el periodo de conmutación.

La ganancia del sensor de corriente $R_{i}=0,2 \Omega$.

La ganancia del modulador PWM bipolar $\left(\mathrm{F}_{M}\right)$ se define como:

$$
F_{M}=\frac{1}{V_{p p_{-} \text {Triangular }}}=1
$$

De donde $V_{p p_{\text {Triangular }}}$ es la tensión pico a pico de la señal triangular que representa la moduladora.

El siguiente paso es determinar el controlador de corriente, dicho controlador fue implementado a través de un controlador resonante (o controlador armónico si sus frecuencias son múltiplos de la fundamental) [22]. Dichos controladores tienen la ventaja de introducir en el lazo una alta ganancia a la frecuencia de la señal de consigna y proveer a la salida la contribución necesaria para anular el efecto causado por las perturbaciones de la red eléctrica. Para esta aplicación el controlador empleado fue un $P+$ Resonante.

$$
G_{S}(s)=K_{p}+\frac{K_{h} \cdot B_{h} \cdot s}{s^{2}+B_{h} \cdot s+\omega_{h}^{2}}
$$

De donde $\omega h=h \bullet \omega O$, es la pulsación de resonancia múltiplo del fundamental. $K_{h}$ es la ganancia del pico de resonancia a la frecuencia $\omega_{h}$. $B_{h}$ es el ancho de banda en rad/s donde la resonancia tiene ganancia. Para este caso $h=1, K_{h}=100, B_{h}=2 \cdot \pi y$ $\omega O=2 \cdot \pi \cdot 50$. EL valor de $K_{p}$ puede ser calculado a través de la siguiente ecuación.

$$
K_{p}=\frac{L \cdot \omega_{c_{-} \text {Deseada }}}{R_{i} \cdot F_{M} \cdot 2 \cdot V_{D C}}=1,0446
$$

En la Fig. 10 se muestra el diagrama de bode para el lazo de corriente de la Fig. 7 el cual se define como $T_{i}(s)=G_{i L-d}(s) \cdot F_{M} \cdot R_{i} \cdot G_{s}(s)$ para una $R_{d}$ igual $0 \Omega$ у а $50 \Omega$. En dicha figura se puede observar como en aras de cumplir con la condición de que

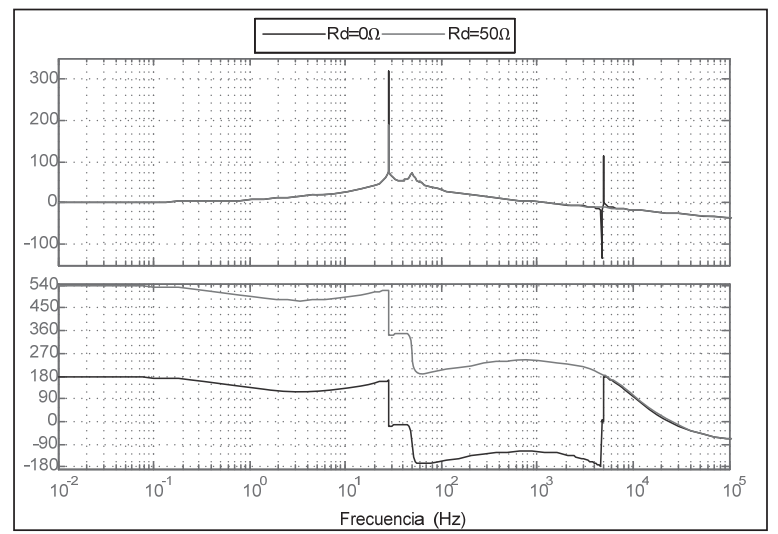

Fig. 10. Diagrama de bode de la función de transferencia de la ganancia del lazo de corriente Ti(s) para $\mathrm{Rd}=0 \Omega$ y $50 \Omega$. 
la amplitud de la señal de resonancia se encuentre por debajo de $0 \mathrm{~dB}$ se ha tomado una resistencia de $50 \Omega$. Adicionalmente se muestra como con el controlador propuesto se logra un margen de fase de 58,60 y un ancho de banda de $1,28 \mathrm{kHz}$.

El siguiente paso es determinar el controlador de tensión $G_{v}(s)$, para lo cual hay que tener en cuenta que la ganancia del sensor de tensión $\beta=6 \cdot 10^{3}$, y la función de transferencia que relaciona la tensión de la DC_LINK a la tensión que impone como referencia el controlador de tensión $G_{V D C-V C}(s)$. Dicha función de transferencia puede ser determinada teniendo en cuenta las funciones halladas en la Ec. (21) y la Ec. (24), y la función auxiliar que relaciona la corriente en el inductor a la tensión de salida del controlador de tensión. Esta función de transferencia se presenta en la Ec. (29)

$$
G_{i_{L}-v_{c}}(s)=\frac{i_{L}}{\hat{v}_{C}}=\frac{T_{i}(s)}{R_{i} \cdot\left(1+T_{i}(s)\right)}
$$

Finalmente la función de transferencia $G_{V D C-V C}(s)$ se define como

$$
G_{v_{D C}-v_{c}}(s)=\frac{\hat{v}_{D C}}{\hat{i}_{L}} \cdot \frac{\hat{i}_{L}}{\hat{v}_{C}}=\frac{\hat{v}_{D C}}{\hat{d}} \cdot\left(\frac{\hat{i}_{L}}{\hat{d}}\right)^{-1} \cdot \frac{\hat{i}_{L}}{\hat{v}_{C}}=\frac{\hat{v}_{D C}}{\hat{v}_{C}}
$$

El controlador de tensión que fija la referencia a seguir por el controlador de corriente y que permite regular la tensión en la DC_LINK, se implementó a través de un controlador PI [23], esto en razón a que dicho controlador permite que el sistema logre error de estado estacionario cero. Por tanto, las salidas reguladas del sistema, tensión o corriente, son exactamente una cantidad específica y permiten seguir la dinámica de la red eléctrica. El controlador implementado se muestra en la Ec. (31).

$$
G_{v}(s)=K_{p}+\frac{K_{i}}{s}=2,5+\frac{4.5}{s}
$$

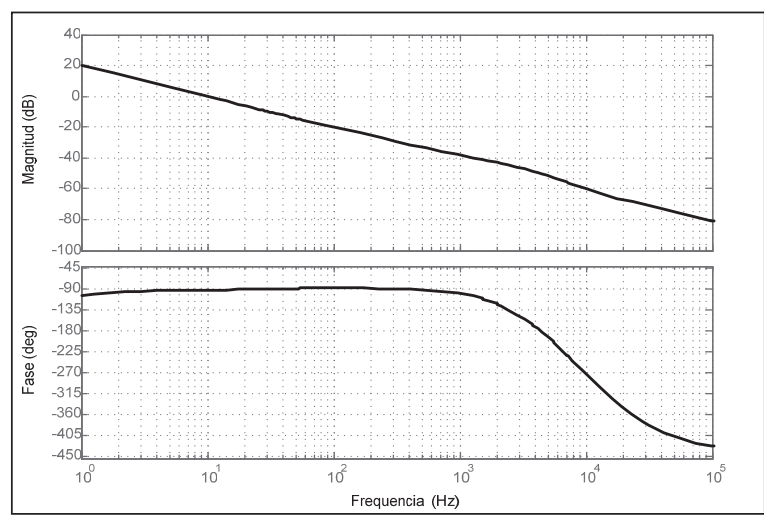

Fig. 11. Diagrama de bode de la función de transferencia de la ganancia del lazo de tensión $\operatorname{Tv}(\mathrm{s})$

En la Fig. 11 se muestra el diagrama de bode para el lazo de tensión de la Fig. 7 el cual se define como $T_{v}(s)=G_{V D C-V c}(s) \bullet \beta \bullet G_{v}(s)$. En el diagrama se muestra que con el controlador propuesto se logra un margen de fase de 85,80 y un ancho de banda de $10 \mathrm{~Hz}$. Dicho valor de frecuencia de corte se ha seleccionado por debajo de la frecuencia del rizado de tensión de la DC_LINK $(100 \mathrm{~Hz})$, con el fin de que dicho rizado esté suficientemente atenuado y no afecte de forma significativa la señal que se inyecta al controlador de tensión.

\subsection{Phase-Locked Loop (PLL)}

Para una correcta operación del control del inversor se hace necesario que el inversor esté adecuadamente sincronizado con la red eléctrica. Esto se puede lograr mediante la utilización de un PLL. Un esquema de PLL generalmente empleado en sistemas trifásicos se basa en el uso de un marco de referencia síncrono (SRF) [24]. Para sistemas monofásicos, la aplicación directa de un SRF, no es posible, pero se puede generar una componente en cuadratura con la señal de tensión a fin de poder aplicar la Transformada Inversa de Park [25]. Una forma de generar dicha componente en cuadratura es implementando un retardo, el cual es el responsable de introducir un desplazamiento de la fase de 


\section{con-ciencias}

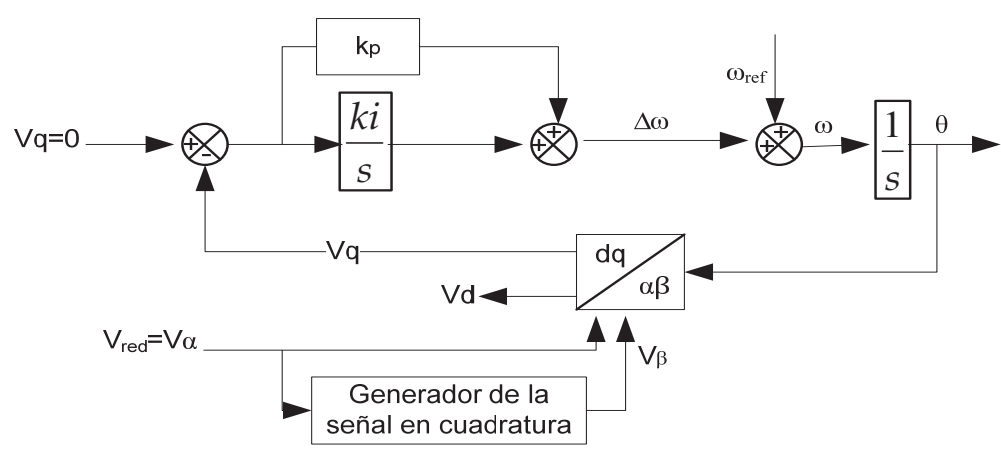

Fig. 12. Algoritmo del PLL monofásico basado en el uso de un retardo para generar la señal en cuadratura.

$90^{\circ}$ con respecto a la frecuencia fundamental de la señal de entrada. En la Fig. 12 se presenta el algoritmo del PLL monofásico basado en el uso de un retardo para generar la señal en cuadratura.

El retardo empleado con el fin de generar el desfase de $90^{\circ}$ puede ser implementado con un filtro paso bajo a una frecuencia de corte mucho menor que la frecuencia de la red $(50 \mathrm{~Hz})$. Esta frecuencia de corte se fijó a $5 \mathrm{~Hz}$. La ganancia de este filtro se escogió en función a la atenuación dada por este, es decir, se busca que las señales $v_{\alpha}$ y $v_{\beta}$ sean iguales, con lo que se obtuvo un valor de 10 .
La respuesta dinámica del PLL, dependerá de los parámetros $K_{p}$ y $K_{i}$, que definirán la frecuencia de corte en el diagrama de bode del lazo cerrado de control. Es común asumir que la respuesta de dinámica del sistema sea estable con un margen de fase superior a $50^{\circ}$, la frecuencia de corte es fijada en función al tiempo de respuesta deseado, para este caso se ha fijado en $25,3 \mathrm{~Hz}$. Los parámetros del regulador PI que proporcionan la respuesta dinámica mostrada en la Fig. 13, son: $K_{p}=149,96$ y $K_{i}=1630$.

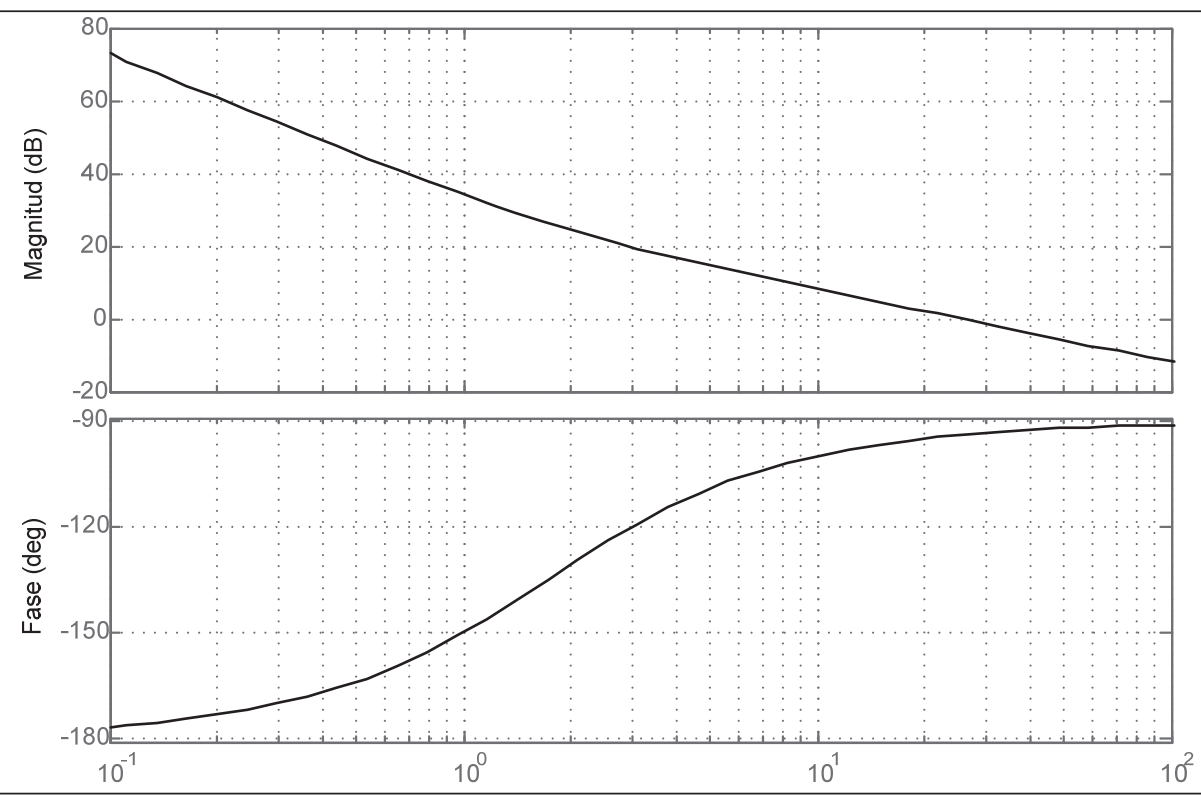

Fig. 13. Diagrama de bode de la respuesta del PLL. 


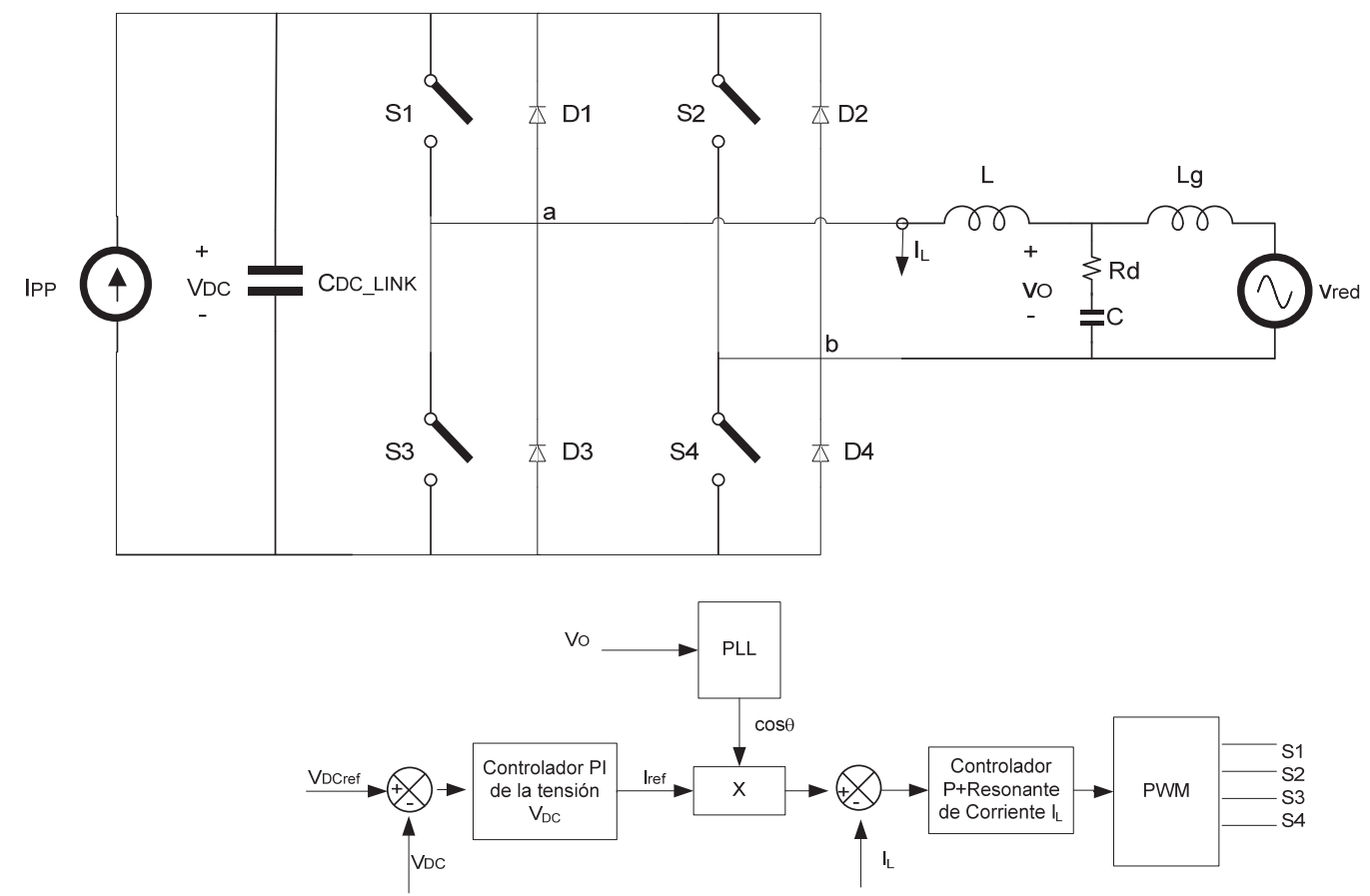

Fig. 14. Esquema del circuito Inversor con control ACC implementado en PSIM 7.05

\section{RESULTADOS DE SIMULACIÓN}

En la Fig. 14 se muestra el esquema circuital del inversor con lazo de control ACC implementado en PSIM 7.05. Para efectos de simplicidad y validación del diseño del inversor, la acción de la fuente primaria sobre el inversor se presenta modelada como una fuente de corriente.

En la Fig. 15 se presentan las principales formas de onda en el inversor interconectado a red, para variaciones de la fuente de corriente que alimenta al inversor.

En la figura anterior se observa que ante variaciones de la corriente de entrada, la corriente de salida se ajusta, permitiendo que se inyecte potencia a la red eléctrica de acuerdo con la energía disponible en la renovable $(430 \mathrm{~W}, 200 \mathrm{~W}, 430 \mathrm{~W}$ y $310 \mathrm{~W})$. Para este rango de variación, la tensión en la DC_LINK permanece constante $(400 \mathrm{~V})$. El va-

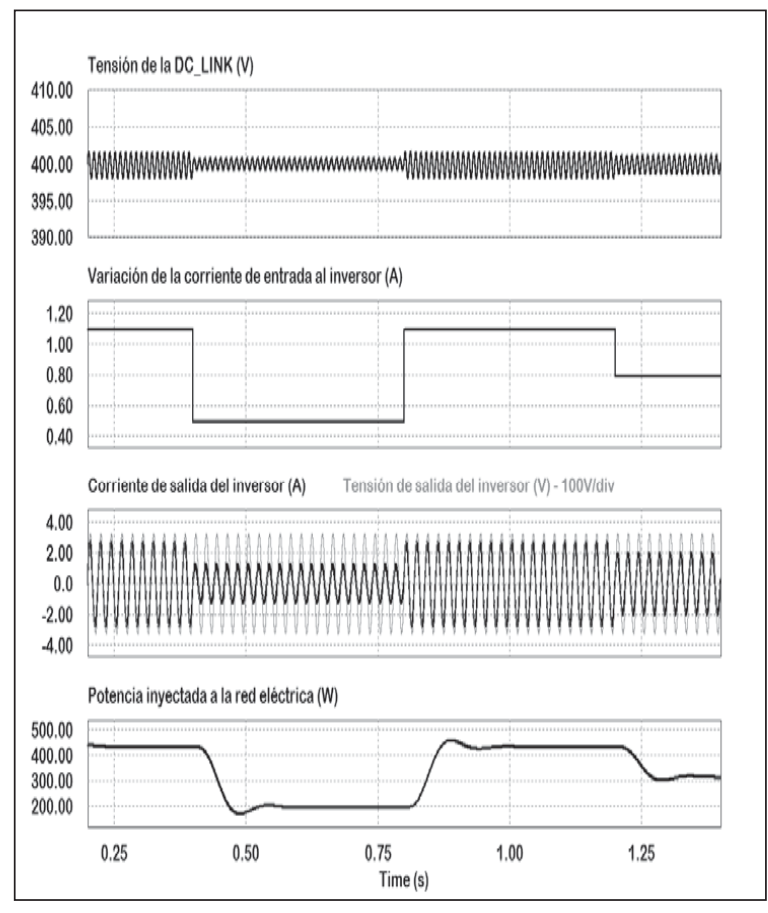

Fig. 15. Principales formas de onda en el inversor interconectado a red. 
lor del THDi de la corriente de salida del inversor, para la potencia nominal (430W) es del 3\%.

\section{RESULTADOS EXPERIMENTALES}

Los resultados experimentales se han llevado a cabo sobre el inversor, con el fin de validar su comportamiento dinámico. Las características del prototipo implementado se presentan en la Tabla 1. El control del inversor ha sido desarrollado a través de la tarjeta de DSP Texas Instruments TMS320F28335.

Tabla 1. Principales parámetros del prototipo inversor

\begin{tabular}{|l|c|}
\hline \multicolumn{2}{|c|}{ Inversor PWM } \\
\hline \multicolumn{1}{|c|}{ Parámetro } & Valor \\
\hline Tensión en la DC_LINK & $380 \mathrm{~V}$ \\
\hline $\begin{array}{l}\text { Máxima potencia de entrada al } \\
\text { inversor }\left(P_{\text {O_PP }}\right)\end{array}$ & $300 \mathrm{~W}$ \\
\hline Tensión de salida $\left(V_{O \text { RMS }}\right)$ & $230 \mathrm{~V}_{\mathrm{RMS}}$ \\
\hline Frecuencia de conmutación $\left(f_{s i}\right)$ & $20 \mathrm{kHz}$ \\
\hline Frecuencia de salida $\left(f_{g}\right)$ & $50 \mathrm{~Hz}$ \\
\hline $\begin{array}{l}\text { Condensador de la DC_LINK } \\
\left(C_{D C \_L I N K}\right)\end{array}$ & $1 \mathrm{mF}$ \\
\hline $\begin{array}{l}\text { Inductor del filtro de salida } \times 2 \\
(L)\end{array}$ & $9.6 \mathrm{mH} \mathrm{a} \mathrm{3.57A}$ \\
\hline $\begin{array}{l}\text { Condensador del filtro de salida } \\
(C)\end{array}$ & $680 \mathrm{nF}$ \\
\hline Resistencia de damping $\left(R_{d}\right)$ & $50 \Omega$ \\
\hline $\begin{array}{l}\text { Interruptores IGBT } \\
\text { IKP20N60T }\end{array}$ \\
\hline
\end{tabular}

Se han realizado dos juegos de pruebas sobre el prototipo, con el objeto de valorar la respuesta transitoria de este. Para las medidas de la respuesta transitoria se ha utilizado una fuente de alimentación programada como fuente de corriente. Cabe resaltar que la fuente de alimentación alimenta directamente al inversor. A continuación se exponen los tipos de pruebas realizados.

\subsection{Pruebas de la respuesta transitoria del inversor ante cambios de la corriente de entrada}

Para estudiar la estabilidad de la respuesta transitoria del inversor se ha configurado la fuente de alimentación DC como una fuente de corriente a la cual se le ha programado escalones de corriente en $I_{p v}$ de $0.4 \mathrm{~A}$ a $0.75 \mathrm{~A}$ y de $0.75 \mathrm{~A}$ a $0.4 \mathrm{~A}$. Cabe resaltar que los escalones de corriente presentan un comportamiento más rápido que los que podrían obtenerse de los paneles fotovoltaicos. Para este caso se ha fijado $V_{D C_{-} \text {ref }}=380 \mathrm{~V}$.

En la Fig. 16 se puede observar la respuesta dinámica de la corriente inyectada a la red, así como de la tensión en la DC_LINK, cuando se generan escalones de corriente en $I_{p v}$. En la Fig. 17 se aprecia una ampliación de las dos variables presentadas en la Fig. 16 cuando se origina un escalón de subida y un escalón de bajada, respectivamente. Como se puede apreciar la respuesta dinámica tanto de la corriente inyectada a la red, así como de la tensión en los bornes del arreglo de paneles fotovoltaicos es estable y ligeramente subamortiguada.

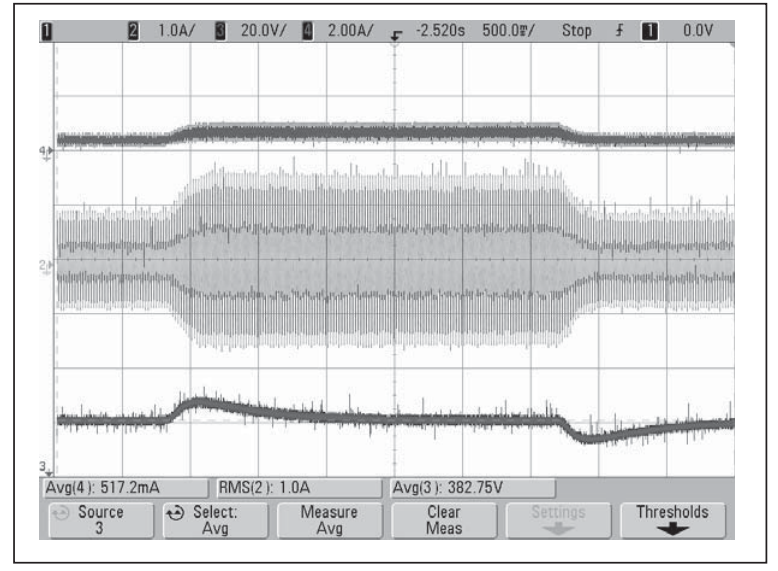

Fig. 16. Escalones de corriente en $\mathrm{I}_{\mathrm{pv}}$, (superior, 2A/ div). Corriente inyectada a la red, (central, 1A/div). Tensión en la DC_LINK, (inferior, 20V/div). Escala de tiempo, 500 ms/div. 


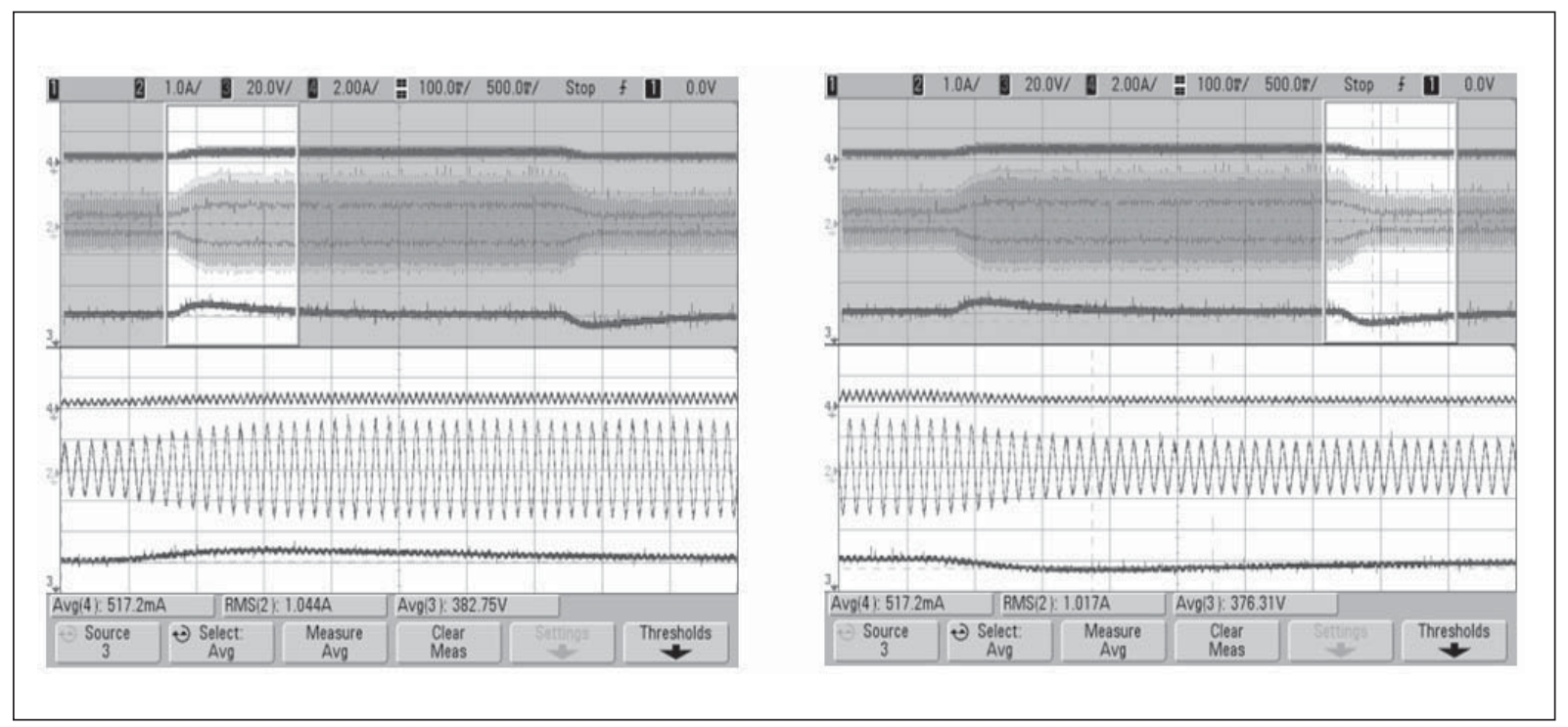

Fig. 17. Detalle de: Escalón de subida (izquierda) y de bajada (derecha) de la corriente en $\mathrm{I}_{p v}$, (superior, $2 \mathrm{~A} / \mathrm{div}$ ). Corriente inyectada a la red, (central, 1A/div). Tensión en en la DC_LINK, (inferior, 20V/div). Escala de tiempo, $500 \mathrm{~ms} /$ div.

\subsection{Distorsión de la corriente de línea del inversor}

El inversor monofásico funciona como rectificador VSI con PWM bipolar, regulando la tensión de la DC_LINK a 380V. El control de corriente del inversor, inyecta a la red la corriente de salida en fase con la tensión de red. La Fig. 18 muestra las medidas experimentales de los armónicos de la corriente inyectada, representados en un diagrama de barras, para una potencia de $300 \mathrm{~W}$ y 150W. Dichos datos fueron tomados con el Fluke 43B Power Quality Analyzer. Se puede observar que a medida que aumenta la potencia inyectada el valor de los armónicos en corriente disminuye, lo que se traduce en una disminución del THD en corriente cuando se inyecta más potencia.

La Fig. 19 muestra la corriente inyectada a red y la tensión de red en el punto de operación $\mathrm{V}_{\text {DCLINK }} \approx 380 \mathrm{~V}, \mathrm{PO} \approx 300 \mathrm{~W}$. Para este punto de operación se tiene una distorsión de la tensión de red ideal, THDV $=0.2 \%$, la distorsión de la corriente inyectada a la red es THDi $=4.5 \%$. En

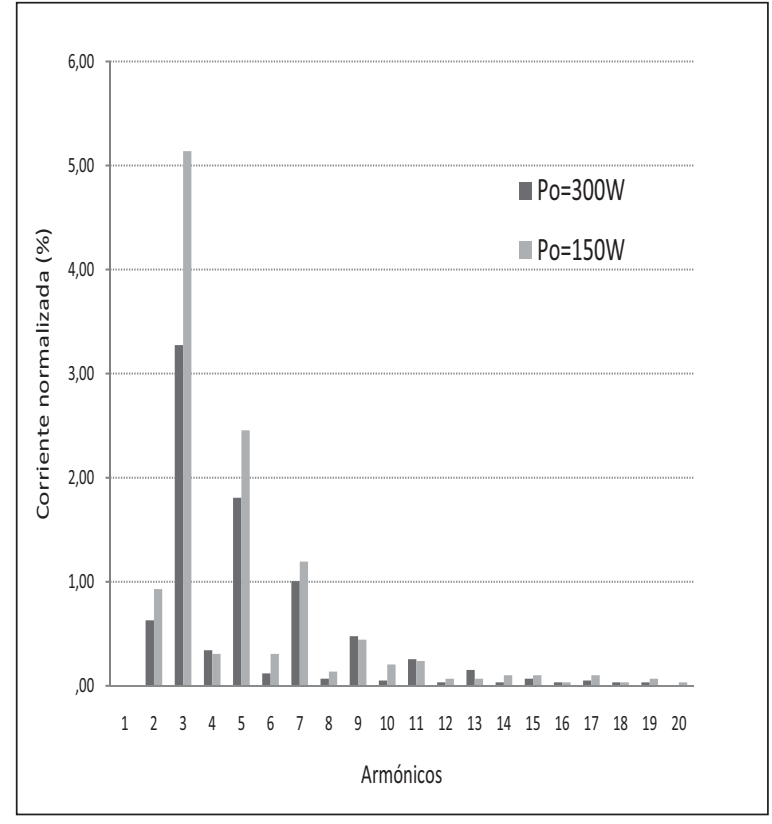

Fig. 18. Diagrama de barras para los armónicos medidos a la corriente normalizada inyectada a la red para 300W (negro) y 150W (gris)

estas condiciones el factor de potencia del inversor es $\mathrm{PF}=0.98$. Según las medidas obtenidas anteriormente es posible inferir que el inversor 


\section{con-ciencias}

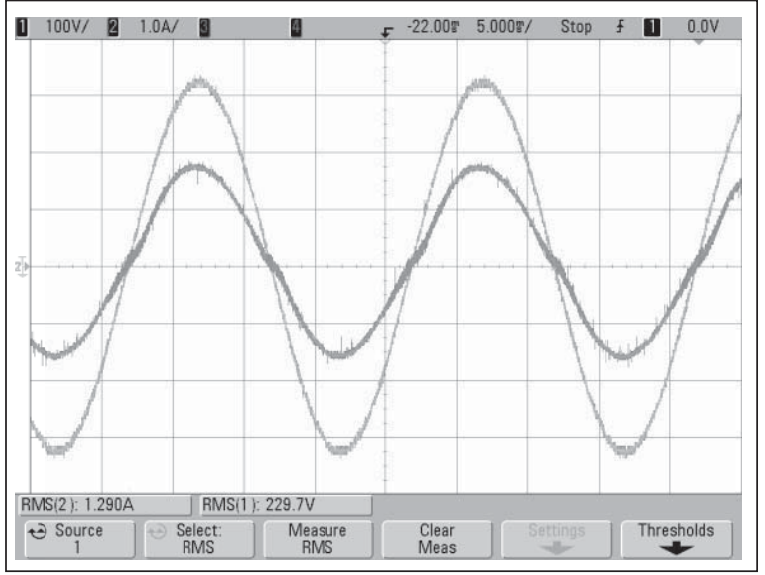

Fig. 19. Respuesta experimental en el punto de operación:VDC_LINK $\approx 380 \mathrm{~V}, \mathrm{PO} \approx 300 \mathrm{~W}$ de, corriente inyectada a la red, (superior, 1A/div). Tensión de red, (inferior, 100V/ div). Escala de tiempo, $5 \mathrm{~ms} / \mathrm{div}$.

cumple con la norma IEC 61000-3-2, válida para dicho nivel de potencia.

\section{CONCLUSIONES}

Se diseñó e implementó un inversor con topología puente completo y control de corriente media (ACC). Dicho esquema de control presenta un lazo interno de control de corriente que permite inyectar la corriente de salida del inversor a la red y un lazo de tensión que fija la referencia del lazo de control de corriente y regula la tensión de la DC_LINK. Los controladores en los diferentes lazos de control fueron obtenidos a través de un modelo en pequeña señal a partir del modelo del conmutador PWM y de la aproximación del inversor a un convertidor Buck.

Finalmente, se validó a través de simulación y de forma experimental la respuesta transitoria y en estado estable del inversor implementado para operar en el modo interconectado a red, obteniéndose resultados adecuados.

\section{REFERENCIAS}

[1] REN21 Steering Committee, Renewable 2009, Global Status Report, Worldwatch Institute Washington, DC, 2009.

[2] J.A. Hernández, D. Velasco and C.L. Trujillo, "Analysis of the Effect of the Implementation of Photovoltaic Systems Like Option of Distributed Generation in Colombia", Renewable and Sustainable Energy Reviews, vol. 15, pp. 2290-2298, Jun. 2011.

[3] O. Carranza, G. Garcerá, E. Figueres and L.G. González, "Peak Current Mode Control of Three-phase Boost Rectifiers in Discontinuous Conduction Mode for Small Wind Power Generators", Applied Energy, vol. 87, pp. 2728-2736, Aug. 2010.
[4] Y. Chen, H. Wu, Y. Chen, K. Lee and S. Shyu, "The AC Line Current Regulation Strategy for the Grid-Connected PV System", IEEE Trans. on Power Electronics, vol. 25, no.1, pp. 209-218, Jan. 2010.

[5] J. Selvaraj and N. A. Rahim, "Multilevel Inverter For Grid-Connected PV System Employing Digital PI Controller", Industrial Electronics, IEEE Transactions, vol. 56, no.1, pp. 149-158, Jan. 2009.

[6] H.R. Karshenas and M. Niroomand, "Design and Implementation of a Single Phase Inverter with Sine Wave Tracking Method for Emergency Power Supply with High Performance Reference", Electrical $M a$ - 


\section{con-ciencias}

chines and Systems, ICEMS 2005, Proceedings of the Eighth International Conference on, vol. 2, pp. 1232-1237, 29 Sept. 2005.

[7] Y. Hu, L. Chang and B. Cao, "Novel Predictive Voltage Controlled UPS Inverter for an Improved Stand-alone wind Turbine System", Electrical and Computer Engineering, CCECE '09, Canadian Conference on, pp. 398-402, 3-6 May. 2009.

[8] S. Mekhilef, N.A. Rahim and A.M. Omar, "A New Solar Energy Conversion Scheme Implemented Using Grid-tied Single Phase Inverter", IENCON 2000, Proceedings, vol. 3, pp. 524-527, 2000.

[9] Y. H. Chung, H. J. Kim, G. H. Kwon, T. B. Park, S. H. Kim, K. S. Kim and J. W. Choe, "Neural Network Controlled Voltage Disturbance Detector and Output Voltage Regulator for Dynamic Voltage Restorer", Power Electronics and Applications, 2007 European Conference on, pp. 1-9, 2-5 Sept. 2007.

[10] D.W. Hart, Introduction to Power Electronics, Prentice Hall, 1997.

[11] N. Mohan, T. Undeland and W. Robbins, Power electronics: converters, applications, and design”, Minnesota: John Wiley \& Sons, 1995.

[12] J.C. Crebier and J.P. Ferrieux, "PFC Full Bridge Rectifiers EMI Modelling and Analysis-common Mode Disturbance Reduction", Power Electronics, IEEE Transactions on, vol. 19, no. 2, pp. 378-387, Mar. 2004.

[13] P.A. Dahono and A. Purwadi, "An LC Filter Design Method for Single-phase PWM Inverters", Power Electronics and Drive
Systems, 1995. Proceedings of 1995 International Conference on, vol. 2, pp. 571576, 21-24, Feb. 1995.

[14] J. Kim, J. Choi and H. Hong, "Output LC Filter Design of Voltage Source Inverter Considering the Performance of Controller", Power System Technology, PowerCon 2000, International Conference on, vol. 3, pp. 1659-1664, 2000.

[15] M. Liserre, F. Blaabjerg and S. Hansen, "Design and Control of an LCL-filter-based three-phase Active Rectifier", Industry Applications, IEEE Transactions on, vol. 41, no. 5, pp. 1281-1291, Sept.- Oct. 2005.

[16] K. H. Ahmed, S. J. Finney and B. W. Williams, "Passive Filter Design for ThreePhase Inverter Interfacing in Distributed Generation", Compatibility in Power Electronics, CPE ‘07, pp. 1-9, May. 29 - Jun. 1. 2007.

[17] S. B. Kjaer, J. K. Pedersen and F. Blaabjerg, "A Review of Single-phase Grid-connected Inverters for Photovoltaic Modules", Industry Applications, IEEE Transactions on, vol. 41, no. 5, pp. 1292-1306, Sept.Oct. 2005.

[18] F. Renken, "The DC-link Capacitor Current in Pulsed Single-phase H-bridge Inverters", Power Electronics and Applications, European Conference on, pp. 10, 2005.

[19] V. Vorperian, "Simplified Analysis of PWM Converters Using Model of PWM Switch. Continuous Conduction Mode", Aerospace and Electronic Systems, IEEE Transactions on, vol. 26, no. 3, pp. 490-496, May. 1990.

[20] D. M. Sable, R. B. Ridley and B. H. Cho, "Comparison of Performance of Singleloop and Current-injection-control for 


\section{con-ciencias}

PWM Converters Which Operate in Both Continuous and Discontinuous Modes of Operation", Power Electronics Specialists Conference, PESC '90 Record, 21st Annual IEEE, pp. 74-79, 11-14 Jun. 1990.

[21] Y.S. Jung, J.Y. Lee and M.J. Youn, "A New Small Signal Modeling of Average Current Mode Control", Power Electronics Specialists Conference, IEEE PESC 1998, vol. 2, pp. 1118-1124, 17-22, May. 1998.

[22] D. N. Zmood and D. G. Holmes, "Stationary Frame Current Regulation of PWM Inverters With Zero Steady-state Error", Power Electronics, IEEE Transactions on, vol. 18, no. 3, pp. 814-822, May. 2003.
[23] B.C. Kuo and F. Golnaraghi, "Automatic Control Systems", Hoboquen: John Wiley \& Sons, 2002.

[24] V. Kaura and V. Blasko, "Operation of a Phase Locked Loop System Under Distorted Utility Conditions", Industry Applications, IEEE Transactions on, vol. 33, no. 1, pp. 58-63, Jan.-Feb. 1997.

[25] S. M. Silva, B. M. Lopes, B. J. Filho, R. P. Campana and W. C. Bosventura, "Performance Evaluation of PLL Algorithms for Single-phase Grid-connected Systems", Industry Applications Conference, 2004. 39th IAS Annual Meeting. Conference Record of the 2004 IEEE, vol. 4, pp. 22592263, 3-7, Oct. 2004. 